\title{
Rosy outlook for people with diabetes
}

Published at www.cmaj.ca on Feb. 10

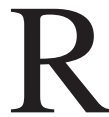
omantics may see the eye as a window into the soul, but doctors tend to view it as a window into the body. The condition of the eye can point not only to ocular diseases but also to more general health problems, such as hypertension. Glucose levels can also be monitored on the eye - in tears - and a Canadian researcher is working on contact lenses to do just that. She hopes her research will save people with diabetes from a lifetime of finger pricks.

"Diabetic patients have to prick their fingers several times a day, and it's quite uncomfortable," says Jin Zhang, assistant professor of chemical and biochemical engineering at the University of Western Ontario in London. "There is a need for a noninvasive measure to detect the level of glucose, instead of taking a blood sample."

Zhang's contact lenses contain nanoparticles that undergo a chemical reaction when exposed to a particular concentration of glucose. The reaction produces a subtle red colour on a lens. "It won't affect people's vision," Zhang says.

She has not tested the contact lenses on human eyes but has successfully tested them in her lab with artificial tears. Zhang has applied for a patent on the technology and has already attracted interest from industry. Her work has also drawn the attention of the Canadian Foundation for Innovation, which recently gave her \$216 342 to further her research. If all goes well, says Zhang, the contact lenses could begin testing in clinical trials in about a year.

Zhang is not the first person to recognize the appeal of a noninvasive test to monitor glucose levels.

Lein Applied Diagnostics Ltd., based in the United Kingdom, is developing a cellphone-sized meter that can be held to the eye to take a glucose measurement. Researchers at Arizona State University in Tempe have created

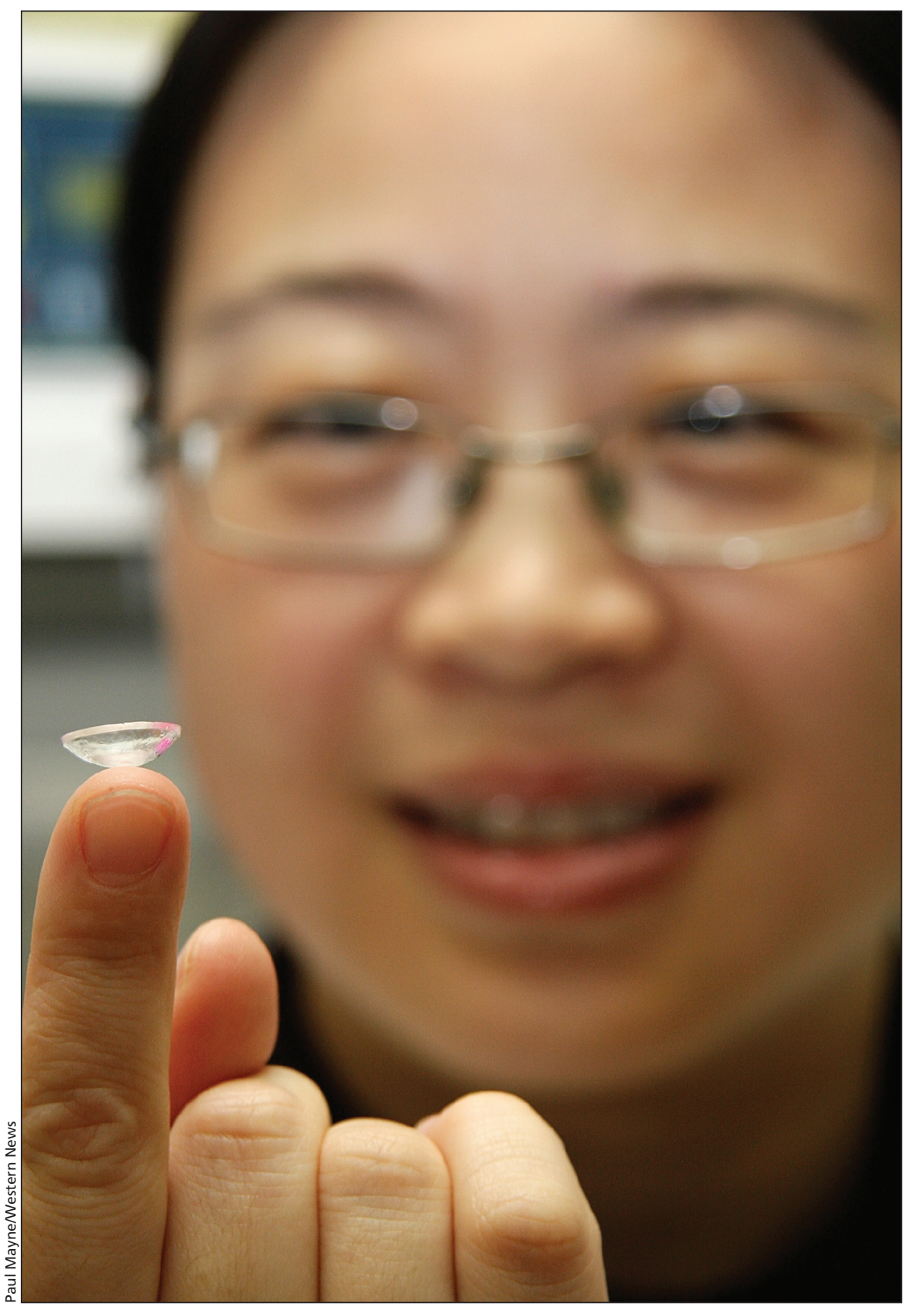

Canadian researcher Jin Zhang is developing contact lenses that change colour according to the concentration of glucose in tears.

a disposable sensor that reacts with tears to produce electricity in amounts that correspond to glucose concentration. At the University of Connecticut in Mansfield, researchers are working on a wireless device - the size of a grain of rice - that will monitor glucose levels for several months after being injected under the skin with a hypodermic needle.

Like Zhang's contact lenses, these technologies are still in early stages of 
development. Still, experts in eye health see potential, especially in the use of nanotechnology.

Dr. Guillermo Rocha, an eye surgeon at the GRMC Vision Centre in Brandon, Manitoba, says it is easy to imagine other applications of nanoparticles in contact lenses. For instance, he says, they could be used to deliver medication, such as antibiotics or anti-inflammatories.

As for monitoring glucose, Rocha recognizes the potential but warns of challenges.
"When you measure glucose in blood, it's a well-controlled environment," he says. "When you are using tear film, there will be variations depending on your environment. It will be different in a humid environment than somewhere dry. These things would have to be taken into account."

And there are other challenges, which Zhang says she is aware of and working to address. These include finding a way to detect more subtle changes in glucose, developing a portable reader to provide actual measurements (as opposed to just indicating change) and solidifying the relationship between glucose levels in tears and the concentration in blood.

"The commercial method of measuring glucose in blood is quite mature," says Zhang, "but measuring glucose in tears is still relatively new." — Roger Collier, CMAJ

DOI:10.1503/cmaj.109-3180 